\title{
Relationships between upper extremity performance and unified Parkinson's disease rating scale-motor exam
}

\author{
Wonjeong Hwang ${ }^{a}$, Sujin Hwang ${ }^{b}$, Kyoungsuk Lee ${ }^{a}$, Yijung Chung ${ }^{c}$ \\ ${ }^{a}$ Department of Physical Therapy, The Graduate School, Sahmyook University, Seoul, Republic of Korea \\ ${ }^{b}$ Department of Physical Therapy, Division of Health Science, Baekseok University, Cheonan, Republic of Korea \\ ${ }^{c}$ Department of Physical Therapy, College of Health and Welfare, Sahmyook University, Seoul, Republic of Korea
}

Objective: Clinical measures that quantify upper extremity function are needed for the accurate evaluation of patients and to plan an intervention strategy. The purpose of this study was to examine the relationship between the Unified Parkinson's Disease Rating Scale (UPDRS)-Motor Exam and upper extremity performance as a quantifying clinical tool of upper extremity function in persons with Parkinson's disease.

Design: Cross-sectional study.

Methods: Thirty-two idiopathic Parkinson's Disease persons participated in this study. To investigate the relationship between the UPDRS-motor exam, Box and Block test (BBT), and Action Research Arm Test (ARAT) by two physical therapists. The examination took up to 1 hour, and the participants were invited to rest between each clinical measure in order to minimize the effects of fatigue. Clinical measures were assessed while the subjects were in the "on" phase of their medication cycle, generally 1-3 hour after taking their anti-Parkinson's medications.

Results: In more affected side, the UPDRS-motor exam was significantly negative correlated with the BBT $(p<0.05)$ but it was not significantly correlated with the ARAT. In less affected side, only positively correlation was significantly shown between BBT and ARAT $(p<0.05)$. On the other hand, between BBT and ARAT were not significantly correlated with the UPDRS-motor exam. Conclusions: The UPDRS-motor exam is effective tool which was significantly correlated with manual dexterity in more affected upper extremity. But The UPDRS-motor exam is not effective tool in less affected upper extremity.

Key Words: Parkinson's disease, Upper extremity, Unified Parkinson disease rating scale

\section{Introduction}

Parkinson's disease (PD) is the most common progressive neurodegenerative disease next to Alzheimers disease, and develops as a result of loss of dopaminergic neuronal degeneration in the basal ganglia, especially the substantia nigra [1]. Individuals with PD may experience motor and cognitive impairment, such as slowness of movement, stiffness, episodes of freezing, and problems with memory and executive functioning $[2,3]$. These deficits can interfere with functional activities such as gait performance, writing, turning around, postural stability and transfer to other positions. Therefore, PD populations tend to be less active, and may provide evidence for the continued loss of independence in performance of daily activities and quality-of-life due to progression of motor deficits [4].

Due to the slowness of movement, many people with PD compromise the functional abilities of upper extremity to reaching, grasping, and manipulating objects. They may also generate abnormally high grip forces when performing precision grip tasks such as lifting a peg or a pencil, and a scaling disorder which can impede sequential tasks during

Received: 27 September, 2013 Revised: 30 November, 2013 Accepted: 9 December, 2013

Corresponding author: Yijung Chung

Department of Physical Therapy, College of Health and Welfare, Sahmyook University, 815 Hwarang-ro, Nowon-gu, Seoul 139-742, Republic of Korea Tel: 82-2-3399-1637 Fax: 82-2-3399-1639 E-mail: yijung36@syu.ac.kr

(c) This is an Open-Access article distributed under the terms of the Creative Commons Attribution Non-Commercial License (http://creativecommons.org/licens es/by-nc/3.0) which permits unrestricted non-commercial use, distribution, and reproduction in any medium, provided the original work is properly cited.

Copyright $@ 2013$ Korean Academy of Physical Therapy Rehabilitation Science 
performance of daily activities, such as grooming, dressing, eating, and writing [5]. Clinical characteristics of PD may be explained by outcome measures that provide a simple and comfortable method for identification and assessment of changes in functional activities and effects of therapeutic intervention.

The Unified Parkinson's Disease Rating Scale (UPDRS) is used most widely and frequently for research and clinical practice to monitor psychological and physical aspects, and may apply comprehensive coverage of PD-related symptoms over time $[1,6]$. Although the UPDRS is the most common clinical assessment tool used in the measurement of functional abilities of PD patients, it presents some shortcomings that limit the predictive usefulness of functional abilities of the upper extremity, especially hand performance. In addition, items involving upper extremity performance in the UPDRS are five items used in the activities of daily living (ADL) section (e.g., handwriting, cutting food and handling utensils, dressing, hygiene, turning in bed and adjusting bed clothes) and four items in the motor examination section (e.g., action or postural tremor of hands, finger taps, hand movements, and rapid alternating movement of hands) [1]. These items may be insufficient for measurement of manipulation of objects and table activities using upper extremity. To examine the ability of PD patients using upper extremity in daily activities and work-related environments, the clinical measures need to involve repeated performance of a variety of functional activities.

The purpose of this study was to propose the feasibility of clinical measures in hand and arm performance of PD patients. We compared clinical measures with the UPDRSmotor exam section because physical therapists may find the section particularly useful.

Table 1. Common characteristic of study participants $(\mathrm{N}=32)$

\begin{tabular}{lc}
\hline \multicolumn{1}{c}{ Variable } & Participant \\
\hline Sex (male/female) & $18 / 14$ \\
Age (y) & $75.97(8.50)$ \\
Height $(\mathrm{cm})$ & $161.41(9.42)$ \\
Weight $(\mathrm{kg})$ & $59.34(9.99)$ \\
Dominant side (right/lift) & $23 / 9$ \\
Hoehn \& Yahr stage (2/3) & $23 / 9$ \\
Expected duration (mo) & $30.41(6.49)$ \\
\hline
\end{tabular}

Values are presented as $\mathrm{n}$ or mean (SD).

\section{Methods}

\section{Subjects}

Thirty-two idiopathic PD persons participated in this study. General characteristics of subjects are shown in Table 1. The following inclusion criteria were used: a medically confirmed diagnosis of idiopathic PD. Patients were excluded from the study if they had a history without any other known neurologic or orthopedic disorders that would affect their functional activities, or severe cognitive deficits that precluded support based on the procedures of this study. Data collection was performed in the outpatient physical therapy department of $\mathrm{H}$ rehabilitation center in Seoul and $\mathrm{G}$ rehabilitation center in Gyeonggi.

\section{Procedures}

Clinical measurements were performed using the UPDRSmotor exam, box and block test (BBT), and action research arm test (ARAT) by two physical therapists to investigate the relationship between the UPDRS-motor exam and upper extremity performance. The participants were given the standard verbal instructions for the clinical measures in a quiet and well-organized therapy. The examination took up to 1 hour, and the participants were invited to rest between each clinical measure in order to minimize the effects of fatigue. The raters scored their own judgment of each participant without any discussion and watched the participant performing. The raters have more than 10 years of experience in evaluation of persons with neurological problems by clinical measures, and have previously used the UPDRSmotor exam in PD populations. Clinical measures were assessed while the subjects were in the "on" phase of their medication cycle, generally 1-3 hour after taking their anti-Parkinson's medications [2].

\section{Clinical measurements}

To investigate the correlation of the UPDRS-motor exam for PD patients, three clinical measures involving the UPDRS-motor exam, ARAT, and BBT were evaluated by clinical observation and testing. The UPDRS developed by Fahn et al. [7] has become the "gold standard" because it contains measures across the enablement/disablement spectrum and demonstrates excellent reliability [1,3]. The UPDRS consists of four sections; I-Mentation, Behavior, and Mood (4 items); II-ADL (13 items); III-MOTOR Examination (14 items); IV-Complications of Therapy (11 items) [4]. All sections except IV are scored using a 5-point 
Table 2. Mean and standard deviation of the scores of clinical measures

$(\mathrm{N}=32)$

\begin{tabular}{lccc}
\hline \multicolumn{1}{c}{ Tool } & Mean (SD) & Minimum & Maximum \\
\hline Less affected side & & & \\
Box and block test & $39.84(3.80)$ & 29 & 45 \\
UPDRS-motor exam & $17.97(4.89)$ & 7 & 29 \\
Action research arm test & $46.84(5.66)$ & 35 & 57 \\
More affected side & & & \\
Box and block test & $35.06(4.54)$ & 24 & 43 \\
UPDRS-motor exam & $17.97(3.87)$ & 10 & 26 \\
Action research arm test & $41.63(6.59)$ & 31 & 55 \\
\hline
\end{tabular}

Values are presented as $\mathrm{n}$ or mean (SD).

UPDRS-motor exam: Unified Parkinson's Disease Rating Scalemotor exam.

scale ranging from 0 (no impairment) to 4 (marked impairment). The UPDRS-motor exam was found to have excellent test-retest reliability (intraclass correlation coefficient, 0.90) in patients with PD [5].

The ARAT was devised for evaluation of specific changes in upper limb function after cortical damage. The test consists of 19 tasks grouped into four subtests: grasp, grip, pinch, and gross movement. The performance of each task is rated on a 4-point scale ranging from 0 (no movement possible) to 3 (movement performed normally), with a maximum obtainable score of $57[6,8]$. The test has reported evidence of predictive validity and reliability in patients receiving stroke rehabilitation [9].

The BBT was originally developed for evaluation of gross manual dexterity of adults with cerebral palsy and is used for evaluation of physically handicapped individuals. The test consists of moving, one by one, as many small wooden blocks as possible from one compartment of a box to another within 60 seconds [10]. The test was reported to have a very high inter-rater and test-retest reliability (ICC and rho for main variables $>0.95)$ [11].

\section{Statistical analysis}

This study divided the measured data into the less or more affected side according to functional activities and symptom appearance in extremities. This study measured Cronbach's alpha for internal consistency of the measured data. Pearson's correlations were used in quantification of bivariate associations between the UPDRS-motor exam and other measures (ARAT and BBT). All statistical analyses were performed using PASW Statistics 18.0 (IBM Co., Armonk, NY, USA). Results were considered statistically significant at $p$-value of less than 0.05 .
Table 3. Correlation of upper extremity performance and UPDRS-motor exam in less affected side $(\mathrm{N}=32)$

\begin{tabular}{lrrr}
\hline \multicolumn{1}{c}{ Variable } & BBT & $\begin{array}{c}\text { UPDRS-motor } \\
\text { exam }\end{array}$ & ARAT \\
\hline BBT & & -0.160 & $0.525^{*}$ \\
UPDRS-motor exam & -0.160 & & -0.140 \\
ARAT & $0.525^{*}$ & -0.140 & \\
\hline
\end{tabular}

UPDRS-motor exam: Unified Parkinson's Disease Rating Scalemotor exam, BBT: box and block test, ARAT: action research arm test.

${ }^{*} p<0.05$.

Table 4. Correlation of upper extremity performance and UPDRS-motor exam in more affected side $(\mathrm{N}=32)$

\begin{tabular}{lrrr}
\hline \multicolumn{1}{c}{ Variable } & BBT & $\begin{array}{c}\text { UPDRS-motor } \\
\text { exam }\end{array}$ & ARAT \\
\hline BBT & & $-0.539^{*}$ & $0.703^{*}$ \\
UPDRS-motor exam & $-0.539^{*}$ & & -0.217 \\
ARAT & $0.703^{*}$ & -0.217 & \\
\hline
\end{tabular}

UPDRS-motor exam: Unified Parkinson's Disease Rating Scalemotor exam, BBT: box and block test, ARAT: action research arm test.

${ }^{*} p 0.05$.

\section{Results}

The value of Cronbach's alpha was 0.562 . The mean scores for the UPDRS-motor exam, ARAT, and BBT are shown in Table 2. None of the evaluation scales showed a floor or ceiling effect. On the less affected side, the scores for the BBT, UPDRS-motor exam, and ARAT were 39.84, 17.97, and 46.84, respectively. On the more affected side, the scores for the BBT, UPDRS-motor exam, and ARAT were $35.06,17.97$, and 41.63 , respectively.

Among the 32 participants, BBT showed significant positive correlation with the ARAT $(r=0.525)$ on the less affected side $(p<0.05)$. However, BBT and ARAT did not show significant correlation with the UPDRS-motor exam. Table 3 shows the relationships between the clinical measures on the less affected side. On the more affected side, UPDRS-motor exam showed significant negative correlation with the BBT $(\mathrm{r}=-0.539 ; p<0.05)$, whereas it did not show significant correlation with the ARAT. The BBT also showed significant positive correlation with the ARAT $(\mathrm{r}=0.703 ; p<0.05)$. Table 4 shows the relationship between the clinical measures on the more affected side. 


\section{Discussion}

This study was conducted in order to examine the feasibilities of clinical measures in hand and arm performance in individuals with idiopathic PD. The correlations between UPDRS-motor exam and BBT in the more affected side were investigated. However, the UPDRS-motor exam did not show a significant correlation with the ARAT. Correlation was observed between the BBT and the ARAT on both upper extremities.

Assessments should involve a previously established valid and reliable means for use by physical therapists in determining the effect and outcome of provided or providing a treatment and to determine the process and clinical characteristic of underlying disease for planning an appropriate treatment [1]. Reliable and valid measurements are also invaluable in both research and clinical settings for use in determination of the patient's disease status [12]. Therefore, selection of the assessment tools depends on the basic needs in clinical and research fields. The UPDRS is the most common clinical assessment tool used for evaluation of disease severity and has become the 'gold standard' because it contains measures across the enablement/disablement spectrum such as body structure and function, activity, and participation, and demonstrates excellent reliability [13]. However, due to the broad range of symptoms involved following PD, one of the major progressive neurodegenerative diseases, there is currently no single measure for comprehensive assessment of the full spectrum of disease manifestations. Therefore, continuous introduction and development for accurate and appropriate measurement of symptoms following PD is necessary. Clinicians who treat the individuals with PD, can evaluate outcomes using a range of assessment strategies such as elementary examination as well as measurement of functional activities, ADL, and quality of life.

Previous studies have reported that the UPDRS total score is used routinely in prediction of functional capabilities. Limited data support its predictive usefulness in persons with early-stage PD or across functional tasks [4]. There is insufficient evidence of the relationship between the UPDRS and upper extremity function for PD. This study was conducted in order to investigate the correlation between the UPDRS-motor exam and upper extremity function, such as manual dexterity, grasp, grip, pinch, and gross movement. In the more affected upper extremity, the UPDRS-motor exam showed to have a correlation with manual dexterity only but not with grasp, grip, pinch, or gross movement. In a recent report, it was suggested that the UPDRS does not reflect a comprehensive measure of mobility even in persons with more advanced PD. The findings are parallel to respect relating to walking performances and ADL function in other populations with central nervous system impairment [3]. The reason for the result showing no significant correlation between the UPDRS-motor exam and ARAT would be the specificity of each measurement. The test performance on the UPDRS-motor exam is based on single-repetition trials as well as optimal functional capability including speech, facial expression, tremor at rest, action or postural tremor of hands, rigidity, finger taps, hand movements, rapid alternating movement of hands, leg agility, arising from chair, posture, gait, postural stability, and body bradykinesia and hypokinesia [14]. However, the ARAT is based on a single trial, including grasp, grip, pinch, and gross movements [6].

This study was conducted in order to investigate the relationship between the UPDRS-motor exam and upper extremity performance in individuals with idiopathic PD. The UPDRS-motor exam showed to have a significant correlation with manual dexterity in the more affected upper extremity, whereas the tool did not show a significant correlation with manual dexterity in the less affected upper extremity and ARAT in both upper extremities. This is because our potential limitation of the study was a relatively small sample. The results of this study cannot necessarily be generalized to rehabilitation of more chronic and severe states of PD, because all of the participants had Hoehn and Yahr stages II to III PD. In future studies, ADL and quality of life will be measured for investigation of relationship with the UPDRS-motor exam.

\section{References}

1. Trail M, Protas E, Lai EC. Neurorehabilitation in Parkinson's disease: an evidence-based treatment model. Thorofare (NJ): SLACK Inc.; 2008.

2. Nunnally JC, Bernstein IH. Psychometric theory. 3rd ed. New York: McGraw-Hill; 1994.

3. Brusse KJ, Zimdars S, Zalewski KR, Steffen TM. Testing functional performance in people with Parkinson disease. Phys Ther $2005 ; 85: 134-41$

4. Song J, Fisher BE, Petzinger G, Wu A, Gordon J, Salem GJ. The relationships between the unified Parkinson's disease rating scale and lower extremity functional performance in persons with early-stage Parkinson's disease. Neurorehabil Neural Repair 2009;23:657-61.

5. Siderowf A, McDermott M, Kieburtz K, Blindauer K, Plumb S, Shoulson I; Parkinson Study Group. Test-retest reliability of the 
unified Parkinson's disease rating scale in patients with early Parkinson's disease: results from a multicenter clinical trial. Mov Disord 2002; 17:758-63.

6. Lyle RC. A performance test for assessment of upper limb function in physical rehabilitation treatment and research. Int $\mathrm{J}$ Rehabil Res 1981;4:483-92.

7. Fahn S, Elton R, Members of the UPDRS Development Committee. In: Fahn S, Marsden CD, Calne DB, Goldstein M, editors. Recent developments in Parkinson's disease. Vol 2. Florham Park (NJ): Macmillan Health Care Information; 1987. p 153-163, 293-304.

8. Yozbatiran N, Der-Yeghiaian L, Cramer SC. A standardized approach to performing the action research arm test. Neurorehabil Neural Repair 2008;22:78-90.

9. Chen HF, Lin KC, Wu CY, Chen CL. Rasch validation and predictive validity of the action research arm test in patients receiving stroke rehabilitation. Arch Phys Med Rehabil 2012;93:103945 .
10. Mathiowetz V, Volland G, Kashman N, Weber K. Adult norms for the Box and Block Test of manual dexterity. Am J Occup Ther 1985;39:386-91.

11. Platz T, Pinkowski C, van Wijck F, Kim IH, di Bella P, Johnson G. Reliability and validity of arm function assessment with standardized guidelines for the Fugl-Meyer Test, Action Research Arm Test and Box and Block Test: a multicentre study. Clin Rehabil 2005;19:404-11.

12. Portney LG, Watkins MP. Foundations of clinical research: applications to practice. 3rd ed. Upper Saddle River (NJ): Pearson/ Prentice Hall; 2009.

13. Fahn S. Description of Parkinson's disease as a clinical syndrome. Ann N Y Acad Sci 2003;991:1-14.

14. Martinez-Martin P, Chaudhuri KR, Rojo-Abuin JM, RodriguezBlazquez C, Alvarez-Sanchez M, Arakaki T, et al. Assessing the non-motor symptoms of Parkinson's disease: MDS-UPDRS and NMS Scale. Eur J Neurol 2013. [Epub ahead of print] 